\title{
Philosophiques
}

\section{Platon, La République, introduction, traduction et notes de Georges Leroux, Paris, Flammarion, 802 p.}

\section{Benoît Castelnérac}

Volume 29, numéro 2, automne 2002

La démocratie délibérative

URI : https://id.erudit.org/iderudit/006313ar

DOI : https://doi.org/10.7202/006313ar

Aller au sommaire du numéro

Éditeur(s)

Société de philosophie du Québec

ISSN

0316-2923 (imprimé)

1492-1391 (numérique)

Découvrir la revue

Citer ce compte rendu

Castelnérac, B. (2002). Compte rendu de [Platon, La République, introduction, traduction et notes de Georges Leroux, Paris, Flammarion, 802 p.]

Philosophiques, 29(2), 407-411. https://doi.org/10.7202/006313ar d'utilisation que vous pouvez consulter en ligne.

https://apropos.erudit.org/fr/usagers/politique-dutilisation/ 
La phénoménologie de $M$ ichel $H$ enry est une philosophie qui cherche un fondement dans l'intériorité du sujet à partir du sentiment comme donné absolu, autonome, libre de se mouvoir lui-même. Pour cela, une sorte de « réduction » doit être accomplie qui part de l'extériorité pour aboutir à l'immanence du sujet auto-affecté. L'Être est donné «absolument» dans cette intériorité et elle seule peut acceuillirreceuillir le phénomène dans sa manifestation.

Comble de l'audace aux yeux d'un heideggerien, $\mathrm{H}$ enry ajoute un accent résolument «théologique» à sa philosophie de l'auto-donation du sentiment comme révélateur de l'Être du monde; cet Absolu présent dans l'immanence du sujet n'est nul autre que Dieu (le Dieu de M aître Eckhart) révélé par la parfaite correspondance entre le sentiment de l'Être et sa manifestation dans le phénomène donné au sujet égologique. La subjectivité auto-donatrice est le lieu de rencontre entre Dieu et l'H omme, où le sentiment révèle le statut ontologique de la divinité dont l'existence s'identifie avec l'autonomie de l'émotion productrice de tonal ité affective. II n'y a que Dieu qui puisse faire en sorte que l'intériorité du sujet soit indépendante des conditions extérieures de son avènement et il n'y a que Dieu qui puisse lui-même être aussi libre de ces mêmes conditions.

Le livre de $M$ ichel $H$ aar, $L$ a philosophie française entre phénoménologie et métaphysique, constitue ainsi, dans son ensemble, une étude du rapport « polémique» qu'entretient la philosophie française contemporaine avec des philosophes allemands tels $\mathrm{H}$ eidegger et $\mathrm{N}$ ietzsche. Le chapitre sur « Sartre contre H eidegger » est particulièrement intéressant et démontre une bonne compréhension des grandes thèses sartriennes dans leur relation problématique avec la philosophie « existentiale» (et non «existentielle») de H eidegger. Le but de l'ouvrage n'étant pas de fournir une vue d'ensemble sur chaque auteur mais bien de discuter des problématiques particulières, il faut déjà être à l'aise avec le style phénoménologique et avoir une connaissance générale des philosophes abordés pour tirer profit de cette lecture qui laisse deviner, pour sa part, une bonne maîtrise de la phénoménologie allemande et française par l'auteur lui-même.

MARIO CHARLAND

Université du Québec à Trois-Rivières

\section{Platon, La République, introduction, traduction et notes de Georges Leroux, Paris, Flammarion, $802 \mathrm{p}$.}

Depuis la fin des années quatre-vingt, la collection G.-F. propose au grand public et aux étudiants une mise à jour complète des traductions de Platon; la plupart des dialogues sont déjà disponibles, entièrement retraduits et accompagnés d'une introduction et de notes faisant état de l'actualité des recherches sur Platon. Un petit nombre reste encore à paraître (dont l'immense Lois), mais la parution de La République (février 2002) comble un manque d'importance dans cette entreprise de renouvellement. La difficulté était de taille : faire tenir en format poche un commentaire, une traduction et des notes actualisant la pierre angulaire du système platonicien. G. Leroux l'a surmontée, d'une part grâce à un impressionnant travail de synthèse dans l'introduction, d'autre part par un appareil de notes pratiques, faisant ainsi l'économie d'une glose qui, sans des renvois précis à la littérature secondaire et aux dialogues de Platon, 


\section{8 · Philosophiques / Automne 2002}

aurait été trop abondante. L'introduction mérite un commentaire étendu, car elle est captivante et détermine l'ensemble de cette édition.

La collection G.-F. avait habitué les lecteurs à des introductions substantielles, voire copieuses. La présente édition rompt avec cette manière de faire, et cela pour d'évidentes raisons de volume (personne n'aurait voulu d'une République en deux tomes). Toutefois, aussi succincte soit-elle (60 pages pour 400 pages de texte), cette introduction mérite pleinement le nom de commentaire. $\mathrm{N}$ on seulement elle situe le texte dans I'histoire et fait voir la place centrale qu'il occupe dans les œuvres de Platon, ce qu'on est en droit d'attendre d'une introduction, mais outre cela elle élucide la portée du dialogue par une analyse profonde et personnelle de son contenu et de son plan, ce qui confère à cette introduction la qualité principale du commentaire : donner à voir le sens d'une œuvre, et le faire comprendre.

Pour G. Leroux, commenter La République, c'est refuser une «position trop courante aujourd'hui, la dépolitisation de l'œuvre» (p. 14). Ce refus est conforme au texte de $L$ a R épublique, réunion mouvementée et conflictuelle de deux théories de la justice qui s'opposent violemment dans le décor effervescent et enfiévré de la politique athénienne à la fin du $\mathrm{V}^{\mathrm{e}}$ siècle. Si le débat sur la justice autorise une lecture qui prend «la métaphysique comme foyer principal » (id.), le cadre dramatique du dialogue et ses allusions continuelles à l'histoire politique d'A thènes et de la Grèce classique commandent par ailleurs « une lecture centrée sur l'histoire» (id.). La présentation de G. Leroux renouvelle ces deux lectures, d'abord en insistant sur la portée politique du dialogue, ensuite en proposant un découpage inédit de sa structure.

L'introduction recentre La République dans le cadre qui la détermine le mieux : la philosophie politique. Ce faisant, elle révèle un Platon homme de son temps, soucieux des problèmes politiques de son époque (comme en témoigne la L ettre VII). II s'engage, se révolte, défend ses convictions (p. 13-14) en procédant à « une dramatisation du dialogue philosophique et [à] une illustration de la distance qui sépare l'effort philosophique de l'engagement dans I'histoire» (p. 18). Grâce à une lumineuse mise en contexte socio-politique et dramatique du dialogue, ironie et critique apparaissent à l'horizon de l'œuvre. Une fois soulignée la nature engagée de $L$ a R épublique, il semble impossible d'en donner une lecture uniquement individualiste ou spéculative. Q uoiqu'elle restera toujours célèbre pour l'effort théorétique qui s'y déploie, il est d'emblée nécessaire d'en saisir la teneur polémique : «la priorité du politique par rapport à la psychologie morale a toujours constitué une position argumentée sur la base de l'ensemble de l'œuvre» (p. 56). G. Leroux avance donc deux arguments de fond pour soutenir qu'une lecture individualiste « ne semble guère possible» (id.) : «premièrement l'importance politique du conflit et deuxièmement, la nécessité d'un ordre politique pour réaliser la vertu, et donc la subordination de la moralité à la justice de la cité» (p. 57). Si la justesse du premier argument fondé sur une mise en contexte historique apparaît clairement à la lecture de l'introduction, la défense du second argument supposerait pour sa part un commentaire beaucoup plus vaste. Quelques éléments de la preuve sont rapidement élaborés : I'importance du thème de la dissension politique (stásis, p. 56, 57-58) ou le prologue du Timée qui insiste pour souligner le caractère constitutionnel de La R épublique (cf. Timée 17c). Le plus intéressant d'entre eux envisage le débat dans son ensemble pour montrer que par l'effort spéculatif du philosophe, "une brèche est ouverte dans la réalité qui permet d'y découvrir le véritable ordre politique» (p. 60). «C'est le modèle général de la tripartition politique qui maintient [... ] la psychologie des vertus dans un cadre d'interprétation 
politique» (p. 58) : on comprend alors que la politique, sorte de passage obligé, permet à Platon de trouver le moyen de révéler le paradigme de la justice valant également pour l'âme (p. 59).

La seconde originalité de ce commentaire se constate dans le plan qu'il donne de L a R épublique (p. 25-42). G. Leroux propose de diviser le dialogue en huit thèmes organisés autour d'un passage «où vient culminer toute la recherche» (p. 27). Ce passage se trouve en R esp. (IV) 427e-445e, section de l'œuvre qu'il nomme «la dialectique de la justice» (id.). Chacun des huit thèmes est exposé en deux parties, une avant "la dialectique de la justice», l'autre située après. Toutes ces parties se répondent exactement en miroir : «la dialectique de la justice» est enchâssée entre les deux parties consacrées au thème des femmes et des enfants (Resp. (IV) 425e-427c, Resp. (V) 449a-461d). La première partie du thème des femmes et des enfants, «la dialectique de la justice» et la deuxième partie du thème des femmes et des enfants forment ensuite un ensemble qui est lui-même précédé et suivi des deux parties du thème «Guerre et paix» (R esp. (III) 414b-(IV)423d, Resp. (V) 461e-471c). Les deux parties du thème «Guerre et paix » sont elles-mêmes encloses dans le thème du «choix des gardiens». Et ainsi de suite huit fois en comptant le thème «mythe et eschatologie» qui ouvre et clôt le dialogue (R esp. (I) 327a-331d, Resp. (X) 608c-621d). O n se trouve ainsi devant une structure en forme de « grande voûte» dont les pièces pointent toutes en direction du morceau culminant, «la dial ectique de la justice». Le recours à cette structure s'est peut-être inspiré de Jacques Brunschwig qui suggérait que $L a$ République aurait aussi bien pu se terminer après le livre IV (D ictionnaire des œuvres politiques, PUF, p. 819-833). En effet, tout le commentaire de G. Leroux s'articule sur la fin du livre IV comme centre logique de l'ensemble de l'œuvre, et réussit à faire se rejoindre "la dialectique de la justice», qui concerne avant tout la nature des vertus dans l'organisation tripartite de l'âme, et la portée politique de l'œuvre qui en forme le cadre et l'architecture.

Toutefois, cette lecture a pour premier inconvénient de laisser dans l'ombre les passages les plus connus du dialogue (et sans doute de tout Platon - est-il encore besoin de les réciter?) : les analogies de la ligne et du soleil et l'allégorie de la caverne. G. Leroux répond que ces parties méritent une explication et des notes séparées (p. 69). O n comprend alors que la mise en lumière de la portée politique de l'œuvre, dans un cadre aussi étroit, impliquait un sacrifice, celui de réduire la présence de ces fameux passages à une seule mention (p. 32-33). Dans certains cas, il ne faut pas trop tenir à la tradition - dans le cas présent, on trouvera aisément les moyens de renouer avec elle. L'occasion de donner une nouvelle vie à l'œuvre majeure de Platon l'a donc emporté sur I'interprétation classique. Ce commentaire, qui trahit par là ses contraintes d'espace, est ainsi à conseiller avec précaution à des lecteurs qui auraient une connaissance mal assurée de la philosophie de Platon. $M$ ais pourtant la collection $G$.-F. s'adresse précisément à ces lecteurs! N ous croyons y voir un pari que G. Leroux, professeur de philosophie, a lancé à G. Leroux, historien de la philosophie. Saluons son courage d'avoir misé sur un Platon engagé au lieu d'un Platon traditionnel et, peut-être, poussiéreux.

Cette lecture a un défaut plus sérieux qui la traverse de bout en bout. On sait combien est problématique la place que Platon réserve aux mythes dans son œuvre en général et dans $L$ a R épublique en particulier. Plusieurs efforts plus ou moins heureux ont été accomplis afin de réconcilier la critique de la poésie au livre $X$ et l'utilisation pédagogique des mythes aux livres II et III. Un exemple parmi tant d'autres : Ie Preface to Plato d'Eric $A$. $H$ avelock qui pose la problématique de manière remarquable. La nou- 


\section{0 - Philosophiques / Automne 2002}

velle édition de La République ne consacre pourtant aucun développement significatif à cette problématique. Les mises en garde de Platon quant aux mensonges véhiculés par les mythes sur les dieux et sa critique des dérèglements causés par la poésie imitative fournissent l'occasion à $G$. Leroux de passer outre ce problème crucial. $M$ ais pourquoi est-il crucial ? Pour de nombreuses raisons qui concernent la logique même du présent commentaire. Tout d'abord, si on ne donne qu'une piètre valeur aux mythes dans La République on confère la même valeur à l'éducation élémentaire qui les utilise à tout propos. Or, si on insiste sur le caractère mensonger des mythes, leur utilisation politique prend les allures d'une gigantesque - et maladroite - duperie idéologique. Le spectre d'un Platon antidémocrate et peu respectueux de la liberté des classes humbles de la cité planerait au-dessus de tout le dialogue; l'aristocratie de son organisation politique apparaîtrait donc sous des dehors totalitaires. Et la structure «en voûte» du plan proposé ne fait rien pour résorber ce problème. En effet, les parties de l'œuvre consacrées au mythe sont renvoyées à sa périphérie, mettant au même niveau les limites intellectuelles de Céphale et l'imposante puissance du mythe eschatologique, ce qui a pour conséquence d'accentuer plus que jamais la rupture entre une politique recourant aux mythes et une éthique rationaliste. On peut douter qu'il était dans les intentions de $G$. Leroux de donner cette tournure aux doctrines politiques exposées dans $L a$ République. Son commentaire va plutôt en sens contraire : serait-il encore possible que l'excellence de la cité serve de modèle à la justice de l'âme? Comment l'éducation des gardiens aurait-elle pu permettre d'en «révéler le paradigme»? (p. 59) II doit en être autrement, si vraiment l'ordre politique est nécessaire à la réalisation de la vertu, si la moralité est subordonnée à la justice de la cité (p. 57).

Q uelques mots pour présenter la traduction et les notes. O $n$ imagine le nombre considérable d'embûches parfois insurmontables qui encombrent le travail du traducteur de La République; il serait difficile de rendre compte des nombreux détails qui caractérisent cette nouvelle traduction qui a bénéficié d'une relecture multiple de la part d'éminents spécialistes. Dans l'ensemble, on peut dire que les choix du traducteur ont suivi trois idées directrices. La première est caractéristique de l'ensemble des traductions de Platon dans la collection G.-F. Elles ont avant tout pour objectif de rendre actuel le texte de Platon en mettant à jour des traductions parfois vieillottes ou difficiles d'accès pour les lecteurs qui ne connaissent pas le grec ancien. La présente traduction ne déroge pas à la règle, et elle le fait en respectant la rigueur logique des passages dont l'argumentation est serrée tout en prenant plus de hauteur et de souplesse lorsque Platon change de style pour adopter une écriture plus étoffée. D'une manière générale, on ne sent pas ou peu l'influence du traducteur qui suit scrupuleusement le texte original. La seconde caractéristique est cependant plus personnelle et participe du refus de dépolitisation de $L$ a R épublique. La traduction en explique la portée politique, préférant, entre deux traductions, celle qui a une connotation politique. Par exemple, en Resp. (II) 369c3-4, pour oíkesis, qui désigne communément I'habitation, la traduction recourt à « fondation», pour sunoikía on trouve « société» au lieu de "vie en commun». II faut remarquer enfin une particularité qui pourra étonner. Le traducteur a inséré entre crochets $(<>)$ des ajouts qu'il juge nécessaires (p. 68). Une cinquantaine d'endroits sont concernés. Plus de la moitié remplacent effectivement des élisions auxquelles les traducteurs suppléent habituellement sans recourir aux crochets. Certains emplois servent cependant à éclairer le texte selon I'interprétation de l'auteur. Par exemple, G. Leroux ajoute systématiquement « et la poésie» après «la musique» afin de rendre manifeste que, pour Platon, la musique 
inclut la poésie. Cette pratique pourrait choquer certains lecteurs puristes qui aiment voir en Platon un écrivain cultivant le goût du mystère et de l'allusion, mais il est par ailleurs certain qu'elle est le résultat d'un louable parti pris de transparence de la part du traducteur. En tout état de cause, elle indique tant aux hellénistes qu'aux autres lecteurs les endroits où ils doivent être vigilants.

Q uant aux notes, elles subissent le même manque d'espace que l'introduction et se réduisent donc à l'essentiel. Pour qu'elles soient aussi complètes que celles des autres éditions de la même collection, qui ajoutent à la traduction un appareil de notes parfois imposant, il aurait fallu en doubler le volume. Compte tenu que cette collection se destine à un large lectorat, il est toutefois concevable qu'elles aient ces proportions sans que cela prête flanc à la critique. G. Leroux leur donne la plus grande utilité en éclairant la traduction par une explication des concepts grecs et en renvoyant aux études les plus profitables sur le sujet. Les liens avec les auteurs classiques et les œuvres de Platon aideront aussi le lecteur peu familier de la littérature grecque à s'y retrouver dans ce texte à l'immense portée intertextuelle. Aux grandes articulations du débat, elles fournissent de plus une explication substantielle de son enjeu et des conséquences des solutions que propose Platon. S'y ajoutent une bibliographie (24 pages), une série de tableaux explicatifs et les index habituels (noms propres, thèmes, et auteurs anciens).

Cette nouvelle République remplit donc entièrement la fonction d'un efficace outil de travail qui tire le meilleur parti d'un espace passablement réduit en offrant une foule de renseignements nécessaires à une compréhension à la fois originale et authentique du chef d'œuvre de Platon.

BEN OÎT CASTELNÉRAC Université de Montréal

\section{Josiane Boulad-Ayoub et François Blanchard, Les grandes figures du monde moderne, Paris, L'H armattan, 2001, 579 p.}

Le collectif publié sous la direction de M adame Boulad-Ayoub et François Blanchard est la publication d'une série d'interventions et de textes produits dans le cadre d'un cours virtuel de la Chaire Unesco-U qam de philosophie. Les textes sont le plus souvent ceux de $\mathrm{M}$ adame Boulad-Ayoub, François Blanchard s'étant chargé plus spécifiquement, mais non exclusivement, des chapitres sur I'histoire des sciences, et les autres textes sont dus à Benoît M ercier, Paul Dumouchel, Sonia Déragon, Benoît Pépin et Jürgen H eizmann.

On nous y propose une promenade à travers une longue période de l'histoire des idées, allant de la Renaissance à la R évolution française. Le choix de ces périodes est justifié par le contenu même du livre : la Renaissance y est présentée comme un moment d'éveil et d'apparition d'une nouvelle vision de l'homme et du monde, et en ce sens comme un point d'origine. La R évolution marque le point d'arrêt de cette période en ce qu'elle représente en quelque sorte l'aboutissement du long chemin de ces idées de l'humain, de la nature, de la religion, à travers quatre siècles au cours desquels s'accomplira le passage de l'humanisme à l'universalisme. Le fil rouge du livre, comme l'indique son titre, sa problématique sous-jacente, est la notion de figure. II s'y passe en effet deux choses :

Les auteurs ont choisi de mettre l'accent sur les hommes, sur la subjectivité des savants, des auteurs, des peintres, au point de vue de la figure, précisément, 